\title{
Mass measurements of neutron-rich isotopes near $N=20$ by in-trap decay with the ISOLTRAP spectrometer
}

\author{
P. Ascher, ${ }^{1, *}$ N. Althubiti, ${ }^{2,3}$ D. Atanasov, ${ }^{4, \dagger}$ K. Blaum, ${ }^{4}$ R. B. Cakirli, ${ }^{5}$ S. Grévy,${ }^{1}$ F. Herfurth,${ }^{6}$ S. Kreim, ${ }^{4}$ D. Lunney, ${ }^{7}$ \\ V. Manea ${ }^{8, \dagger}$ D. Neidherr, ${ }^{6}$ M. Rosenbusch, ${ }^{9}$ L. Schweikhard, ${ }^{10}$ A. Welker, ${ }^{11}$ F. Wienholtz, ${ }^{10}$ R. N. Wolf,,${ }^{4,}$ and K. Zuber ${ }^{11}$ \\ ${ }^{1}$ Centre d'Études Nucléaires de Bordeaux-Gradignan, Gradignan, France \\ ${ }^{2}$ School of Physics and Astronomy, The University of Manchester, Manchester, United Kingdom \\ ${ }^{3}$ Physics Department, Faculty of Science, Jouf University, Aljouf, Saudi Arabia \\ ${ }^{4}$ Max-Planck-Institut für Kernphysik, Heidelberg, Germany \\ ${ }^{5}$ University of Istanbul, Department of Physics 34134, Istanbul, Turkey \\ ${ }^{6}$ GSI Helmholtzzentrum für Schwerionenforschung GmbH, Darmstadt, Germany \\ ${ }^{7}$ CSNSM-IN2P3-CNRS, Université de Paris Sud, Orsay, France \\ ${ }^{8}$ Experimental Physics Department, CERN, Geneva, Switzerland \\ ${ }^{9}$ RIKEN Nishina Center, Wako, Saitama 351-0198, Japan \\ ${ }^{10}$ Institut für Physik, Universität Greifswald, 17487 Greifswald, Germany \\ ${ }^{11}$ Institut für Kern- und Teilchenphysik, Technische Universität Dresden, Germany
}

(Received 28 February 2019; revised manuscript received 21 May 2019; published 8 July 2019)

\begin{abstract}
The masses of ${ }^{34} \mathrm{Si},{ }^{33,34} \mathrm{Mg}$, and ${ }^{34} \mathrm{Al}$ have been measured with the ISOLTRAP Penning-trap spectrometer at ISOLDE/CERN. The results are in agreement with previous mass measurements and uncertainties have been decreased. The mass of ${ }^{34} \mathrm{Al}$ was determined in two configurations, one following direct production by the ISOLDE facility, favoring the $4^{-}$state, expected to be the ground state, second by in-trap decay of ${ }^{34} \mathrm{Mg}$, followed by recoil-ion trapping, favoring the production of the isomeric $1^{+}$state. A position-sensitive detector was used to clean the ToF-ICR resonance. In addition, the mass of the refractory doubly magic ${ }^{34} \mathrm{Si}$ nucleus was directly measured, using the in-trap decay of ${ }^{34} \mathrm{Mg}$, following two sequential recoil-ion captures. The approach, challenges and conclusions are discussed.
\end{abstract}

DOI: 10.1103/PhysRevC.100.014304

\section{INTRODUCTION}

One of the main discoveries concerning exotic nuclei is the so-called island of inversion around $N=20$, discovered by mass measurements that showed an unexpected increase in binding energy for ${ }^{31,32} \mathrm{Na}$ [1]. For the first time the possibility that a magic number might vanish far from stability was raised. In this $N=20$ case, it has then been shown that the attractive character of the tensor part of the monopole interaction $\pi d_{5 / 2}-v d_{3 / 2}$ leads to a less bound $d_{3 / 2}$ orbital energy when removing protons from the $\pi d_{5 / 2}$ orbital [2,3], in turn decreasing the $N=20$ gap and creating a new shell gap at $N=16$. This effect can be observed in Fig. 1, which

*ascher@cenbg.in2p3.fr

${ }^{\dagger}$ Present address: Instituut voor Kern- en Stralingsfysica, Katholieke Universiteit Leuven, B-3001 Leuven, Belgium.

${ }^{\ddagger}$ Present address: ARC Centre of Excellence for Engineered Quantum Systems, School of Physics, The University of Sydney, Australia.

Published by the American Physical Society under the terms of the Creative Commons Attribution 4.0 International license. Further distribution of this work must maintain attribution to the author(s) and the published article's title, journal citation, and DOI. shows the two-neutron separation energies $S_{2 n}=B(Z, N)$ $B(Z, N-2)$, i.e., the binding energy difference between two isotopes having neutron number $N$ and $N-2 . S_{2 n}$ is an indicator of shell-closure evolution and deformation. Here, one observes that the decrease of $S_{2 n}$ between $N=20$ and $N=22$, i.e., how much less the last two neutrons are bound above $N=20$, is smaller for $\mathrm{Mg}$ and $\mathrm{Na}$ than for $\mathrm{Si}$, which is an indication of shell quenching.

This reduced shell gap facilitates neutron excitations to upper $p f$ orbitals, called intruder states. In addition, an increased quadrupole correlation energy [6] lowers these intruder configurations at low excitation energies, even becoming the ground states for some nuclei, forming the so-called island of inversion. Since its discovery, this region has been intensively studied, in order to describe the inversion mechanism and define the region's still unknown boundaries. The ground states of ${ }^{31-34} \mathrm{Mg}$ and ${ }^{30,31} \mathrm{Ne}$ were shown to be highly dominated by intruder configurations [7-10]. ${ }^{31-34} \mathrm{Al}$ isotopes, supposed to be transition isotopes between the spherical $Z=14$ and the deformed $Z=12$, were also studied in detail by decay spectroscopy [11], $\beta$-NMR [12-15], and mass measurements [5,16]. A unique feature of the $S_{2 n}$ values resulting from mass measurements was observed at $N=21$ where the $\mathrm{Al}$ and Mg curves cross (see Fig. 1) [5]. This unexpected behavior triggered dedicated studies to see if ${ }^{34} \mathrm{Al}$ would be in the island 


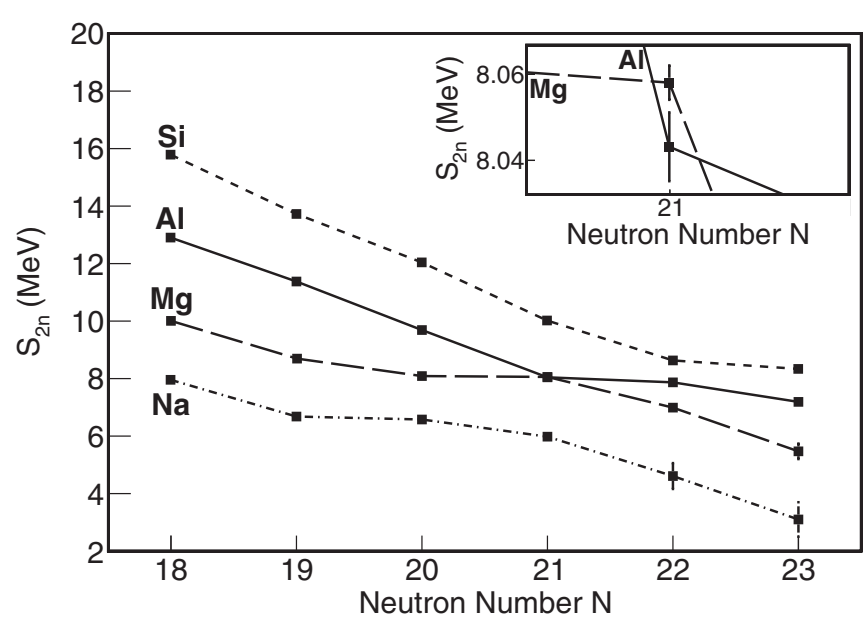

FIG. 1. Two-neutron separation energies $S_{2 n}$ in $\mathrm{MeV}$ (from [4]) for $\mathrm{Si}, \mathrm{Al}, \mathrm{Mg}$, and $\mathrm{Na}$ isotopes. The insert is a zoom on the crossover of $\mathrm{Mg}$ and $\mathrm{Al}$ curves at $N=21$ (see [5]).

of inversion. If this were the case, then the one-particle-onehole $(1 p 1 h)$ intruder state $1^{+}$would be the ground state instead of the $4^{-}$measured in mass measurements, explaining this crossover. However, a recent $\beta$-decay spectroscopy experiment performed at IDS/ISOLDE refuted this hypothesis [17]. Therefore, a direct mass measurement of the $1^{+}$state could help to confirm this assignment. The mass measurement of ${ }^{34} \mathrm{Al}$ was performed recently at TITAN/TRIUMF $[5,16]$ but no sign of the $1^{+}$state could be observed.

$\mathrm{Si}$ isotopes around $N=20$ have also been of high interest $[11,18,19]$, experimentally and theoretically. In particular, ${ }^{34} \mathrm{Si}$ is now a well-established doubly magic nucleus and, as shown recently, the first known bubble nucleus [20,21]. However the mass of this key nucleus was never directly measured.

Penning-trap mass spectrometry is very powerful in terms of accuracy and precision. However, contrary to in-flight (or IGISOL) facilities, access to the properties of exotic nuclei at ISOL facilities strongly depends on the elements' chemistry. In particular, the refractory elements, with very long extraction times from the target, cannot be provided with reasonable yields. This is the case for the element silicon. Different target-ion-source developments are ongoing to solve this problem. Another way to access these elements is to produce them by $\beta$ decay of well-produced isotopes, although the daughter nuclei will be less exotic. If this $\beta$ decay occurs in a gas-filled Penning trap, the decay products can be captured, cooled, and sent to another setup for the measurement. This in-trap decay technique was first implemented using ISOLTRAP [22,23], and allowed to measure the refractory daughter isotopes ${ }^{61-63} \mathrm{Fe}$ from the decays of ${ }^{61-63} \mathrm{Mn}$.

In the present work, a ${ }^{34} \mathrm{Mg}$ beam delivered by ISOLDE allowed to measure the mass of its grand-daughter ${ }^{34} \mathrm{Si}$ isotope, by sequentially capturing the recoil ions ${ }^{34} \mathrm{Al}$ and ${ }^{34} \mathrm{Si}$. Moreover, due to the selection rules of $\beta$ decay, this technique can give access to long-lived isomers that are not produced or extracted from the target. As the $\beta$ decay of ${ }^{34} \mathrm{Mg}$ populates mostly ( $>99 \%)$ the $1^{+}$state in ${ }^{34} \mathrm{Al}$, as shown in Ref. [24],

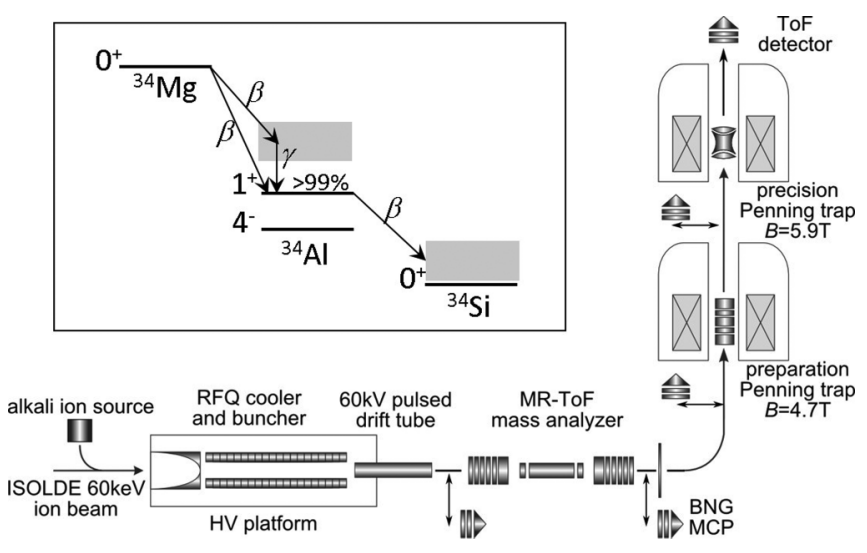

FIG. 2. Sketch of the ISOLTRAP spectrometer consisting of four ion traps: the RFQ cooler-buncher, the MR-ToF component, and the two Penning traps. See text for details. The insert shows a simplified decay scheme of ${ }^{34} \mathrm{Mg}$ (adapted from [17]), populating mostly the $1^{+}$ state in ${ }^{34} \mathrm{Al}$, which further decays to ${ }^{34} \mathrm{Si}$.

the in-trap decay was also used to produce ${ }^{34 m} \mathrm{Al}\left(1^{+}\right)$, of only $26 \mathrm{~ms}$ half-life, the shortest-lived isotope ever attempted with ISOLTRAP. In this article, we report the first Penningtrap mass measurement of the refractory isotope ${ }^{34} \mathrm{Si}$ and the masses of ${ }^{33,34} \mathrm{Mg}$ and ${ }^{34} \mathrm{Al}$ with the mass spectrometer ISOLTRAP [25].

\section{EXPERIMENTAL SETUP AND IN-TRAP DECAY PRINCIPLE}

In the present experiment, the nuclei of interest were produced in two different ways. The ${ }^{34} \mathrm{Mg}$ and ${ }^{34} \mathrm{Al}$ isotopes were delivered by the radioactive ion-beam facility ISOLDE at CERN. To this end, a proton beam of $1.4 \mathrm{GeV}$ was sent on a uranium-carbide target, producing neutral fission products. After extraction from the target, these were laser-ionized by RILIS [26], before being sent to the ISOLTRAP mass spectrometer. A sketch of the setup, consisting of four ion traps, is shown in Fig. 2. More details can be found in Refs. [25,27].

The ions were first trapped for about $5 \mathrm{~ms}$ in the heliumfilled radiofrequency quadrupole (RFQ) and extracted as cooled ion bunches. They were then sent through the electrostatic MR-ToF trap [28] up to the preparation Penning trap. This trap was used to further cool the ions and center the ions of interest, which were then sent to the precision Penning trap, in order to perform the mass measurement via the ToF-ICR method [29,30]. During the present experiment, the MR-ToF component was not used for mass separation [31] nor mass spectrometry [32] due to a short on one of the electrostatic mirrors.

The two other nuclides whose masses are reported in this paper, ${ }^{34 m} \mathrm{Al}$ and ${ }^{34} \mathrm{Si}$, are not directly accessible via the ISOLDE production method. It will be shown in the following that the presence of the aluminium isomer in the ISOLDE beam could not be identified on the last MCP detector of ISOLTRAP. Concerning ${ }^{34} \mathrm{Si}$, silicon is a refractory element, thus has a poor ionization efficiency and a slow release time. However, as shown in the inset of Fig. 2 (adapted from 
TABLE I. Timing patterns of the excitation steps during the trapping in the preparation and precision Penning traps for the nuclides investigated, as compared to the standard values.

\begin{tabular}{lcccr}
\hline \hline & \multicolumn{4}{c}{ Durations (ms) } \\
\cline { 2 - 5 } & $\begin{array}{c}\text { Standard } \\
\text { operation }\end{array}$ & ${ }^{33,34} \mathrm{Mg}$ & ${ }^{34 m} \mathrm{Al}$ & ${ }^{34} \mathrm{Si}$ \\
& & & & \\
\hline Preparation trap & $50-100$ & 25 & 35 & 205 \\
Pre-cooling & 10 & 10 & 10 & 10 \\
Magnetron excitation & $50-200$ & 10 & 10 & 10 \\
Quadrupolar excitation & $5-20$ & 5 & 5 & 5 \\
Cooling of cyclotron motion & 10 & 3 & 3 & 3 \\
Precision trap & $100-1200$ & $25 / 50$ & 20 & 600 \\
Magnetron excitation & \multicolumn{4}{c}{} \\
Quadrupolar excitation &
\end{tabular}

[17]), ${ }^{34} \mathrm{Mg}$ decays mostly to the $1^{+}$state in ${ }^{34} \mathrm{Al}$, which then decays to the ground state of ${ }^{34} \mathrm{Si}$. Therefore, the ${ }^{34} \mathrm{Mg}$ beam produced by ISOLDE was sent to ISOLTRAP, allowing to produce directly in the preparation trap ${ }^{34 m} \mathrm{Al}$ and ${ }^{34} \mathrm{Si}$. Usually, the ions are kept in the gas-filled preparation trap for about $50-100 \mathrm{~ms}$, to prepare the ions before applying the selective buffer-gas cooling technique [33] to remove the contaminations. This so-called precooling time includes a waiting time to axially cool the ions and a short-pulse (broad-band) quadrupolar excitation to center them in the trap. In the present experiment, the short-pulse duration was $5 \mathrm{~ms}$ and the waiting time has been adapted to maximize the rate of ${ }^{34 m} \mathrm{Al}$ or ${ }^{34} \mathrm{Si}$, taking into account the half-lives of ${ }^{34} \mathrm{Mg}\left(T_{1 / 2}=44.9 \mathrm{~ms}\right),{ }^{34 m} \mathrm{Al}\left(T_{1 / 2}=26 \mathrm{~ms}\right)$, and ${ }^{34} \mathrm{Si}\left(T_{1 / 2}=\right.$ $2770 \mathrm{~ms}$ ). The timing sequence of the procedure is listed in Table I. The short half-lives of ${ }^{34 m} \mathrm{Al}$ and ${ }^{34} \mathrm{Mg}$ were a crucial constraint for the minimization of all excitations times. For ${ }^{34} \mathrm{Si}$, the short cycle was not needed but it was easier to keep it the same for the entire beamtime. Only the precooling time mentioned above and the duration of the quadrupolar excitation in the precision trap were adapted to the isotope of interest (see Table I).

Note that whereas the axial trapping potential, the magnetic field, and the damping effect of the buffer gas allow to capture some of the recoiling decay products, not all of them can be kept in the trap. Whether or not the recoil ions are retained depends on both their energy and recoil direction: the ions recoiling axially with an energy higher than the trapping voltage are lost whereas the ions recoiling radially are kept thanks to the strong magnetic field. From the $Q$ value $(Q)$ of the $\beta$ transition, one can calculate the maximum recoiling energy

$$
E_{0}=\frac{Q\left(Q+2 m_{e} c^{2}\right)}{2 m_{\mathrm{recoil}} c^{2}}
$$

of the daughter nuclei [23] in case of a $\beta^{-}$decay, with $m_{e}$ and $m_{\text {recoil }}$ the mass of the electron and the recoiling ion, respectively. In the case of the decay of ${ }^{34} \mathrm{Mg}$ to ${ }^{34} \mathrm{Al}$, the $Q$ value being $11.39 \mathrm{MeV}$, the maximum recoil energy is about $2.3 \mathrm{keV}$. Simulations of the ion motion in a Penning trap were performed with the SIMBUCA program [34]. They showed that if the recoil is in the radial direction, then the maximum energy leads to a radius of $16.8 \mathrm{~mm}$, which is smaller than the trap radius. Then the reduced cyclotron motion is rapidly reduced by the buffer-gas collisions, as well as the magnetron motion thanks to a simultaneous quadrupolar excitation at $v_{c}$ of the ion of interest [33]. The trapping efficiency of the ${ }^{34} \mathrm{Al}$ recoiling ions was estimated to be about $25 \%$. The details of such simulations can be found in Ref. [23], where the influence of the trapping voltage on the capturing efficiency is also discussed. Therefore, for the present measurement, the trapping voltage was increased from the usual $100 \mathrm{~V}$ [25] to $200 \mathrm{~V}$.

These nonstandard conditions in terms of excitation times, endcaps voltages, and, as it will be discussed below, ToFICR scan ranges and steps, were validated by crosscheck measurements of ${ }^{39} \mathrm{~K}^{+}$and ${ }^{85} \mathrm{Rb}^{+}$delivered by the offline ion source.

One should note that for a $\beta$-minus decay, the daughter ions will be doubly charged immediately after the decay (neglecting additional electron shake-off). The first ionization energy of $\mathrm{He}$ being higher than the second one of $\mathrm{Al}$, it was assumed that the level of impurities in the He buffer gas is high enough to transfer most of the ${ }^{34} \mathrm{Al}$ daughter ions to a $1^{+}$charge state after the precooling time. Before the work on ${ }^{34} \mathrm{Al}$, a proof-of-principle test of in-trap decay from ${ }^{33} \mathrm{Mg}$ to ${ }^{33} \mathrm{Al}$ was performed, yielding a production efficiency (including recoil trapping and charge-exchange to the $1^{+}$ionic state, but excluding the decay of the daughter ions) of approximately $5 \%$. Excitation at the frequency of doubly charged ${ }^{34} \mathrm{Al}$ ions was not tried due to time restrictions.

After their production, either by ISOLDE or by decay in the preparation trap, the ions were sent to the precision trap, where the time-of-flight ion cyclotron resonance (ToF-ICR) method was applied to measure their cyclotron frequency $v_{c}$, which is linked to their mass via the relation

$$
v_{c}^{\text {ion }}=\frac{1}{2 \pi} \frac{q B}{m_{\text {ion }}}
$$

with $q$ being the ion charge and $B$ the magnetic-field strength. In the present experiment, the ions were all singly charged, $q$ is then equal to the elementary charge and, assuming that electron binding energies are negligible, $m_{\mathrm{ion}}=m_{a}-m_{e}$ with $m_{a}$ being the atomic mass. Since the magnetic field strength is not precisely enough known to extract the exact mass from Eq. (2), a reference ion with a well-known atomic mass $m_{\text {ref }}$ is used for calibration. At ISOLTRAP such a reference is delivered by an offline alkali ion source, in the present case ${ }^{39} \mathrm{~K}^{+}$. Then, the observable of interest is the ratio $r=v_{c}^{\text {ref }} / \nu_{c}^{\text {ion }}$ and the atomic mass $m_{a}$ of the nuclide of interest is given by

$$
m_{a}=r \cdot\left(m_{\mathrm{ref}}-m_{e}\right)+m_{e} .
$$

$v_{c}^{\text {ref }}$ is linearly interpolated from two measurements right before and after the measurement of the nuclide of interest [35].

\section{RESULTS AND DISCUSSIONS}

The experimental results are summarized in Table II and compared to the AME2016 values [4]. 
TABLE II. Half-lives and frequency ratios of ${ }^{33,34} \mathrm{Mg}^{+},{ }^{34} \mathrm{Si}^{+}$, and ${ }^{34} \mathrm{Al}^{+}$relative to ${ }^{39} \mathrm{~K}^{+}$. The corresponding mass excesses are shown

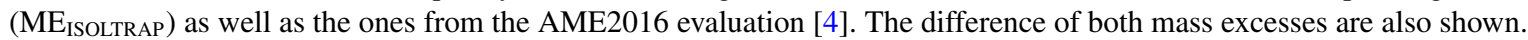

\begin{tabular}{lccccc}
\hline \hline Isotope & Half-life $(\mathrm{ms})$ & $r=v_{c}^{\text {ref }} / \nu_{c}^{\text {ion }}$ & $\mathrm{ME}_{\text {ISOLTRAP }}(\mathrm{keV})$ & ME $_{\text {AME16 }}(\mathrm{keV})$ & $\Delta_{\text {AME-ISOLTRAP }}(\mathrm{keV})$ \\
\hline${ }^{33} \mathrm{Mg}$ & $90.5(1.6)$ & $0.84707669(19)$ & $4966.2(6.8)$ & $4962.3(2.9)$ & $3.9(7.4)$ \\
${ }^{34} \mathrm{Mg}$ & $44.9(0.4)$ & $0.87283446(19)$ & $8323.2(6.9)$ & $8323(29)$ & $0.2(29.8)$ \\
${ }^{34} \mathrm{Si}$ & $2770(200)$ & $0.872054302(22)$ & $-19991.7(0.8)$ & $-19957(14)$ & $34.7(14.0)$ \\
${ }^{34} \mathrm{Al}$ & $56.3(0.5)$ & $0.87252260(8)$ & $-2995.4(2.9)$ & $-3000(3)$ & $4.6(4.2)$ \\
\hline \hline
\end{tabular}

Conventionally, not masses themselves but mass-excess values

$$
\operatorname{ME}(N, Z)=m_{a}-A \mathrm{u}
$$

are compared, where $A$ denotes the mass number and $\mathrm{u}$ the atomic mass unit derived from ${ }^{12} \mathrm{C}$.

$$
\text { A. }{ }^{33,34} \mathrm{Mg}
$$

A ${ }^{33} \mathrm{Mg}$ resonance was measured with an excitation time of $25 \mathrm{~ms}$. As shown in Table II, the resulting mass excess of ${ }^{33} \mathrm{Mg}$ agrees very well with the one of the AME2016 [4]. Two ToF-ICR measurements of ${ }^{34} \mathrm{Mg}$ were performed with an excitation time of $25 \mathrm{~ms}$ and $50 \mathrm{~ms}$, respectively. The obtained mass excess for ${ }^{34} \mathrm{Mg}$ of $8323.2(6.9) \mathrm{keV}$ is also in agreement with the reported AME2016 value [4], mostly based on a measurement performed at TITAN [10]. The mass uncertainty was improved by a factor of four, confirming the large deviation observed with respect to the value of a previous ToF experiment [36]. This result is therefore in agreement with the conclusion of a very weak shell gap $N=$ 20 at $Z=12$, which can be quantified by the empirical shell gap $\Delta_{n}=S_{2 n}(Z, N)-S_{2 n}(Z, N+2)$. For the ${ }^{32} \mathrm{Mg}$ isotope, one can calculate its empirical shell gap using the masses of ${ }^{30,32,34} \mathrm{Mg}$. The new $\Delta_{n}$ value for ${ }^{32} \mathrm{Mg}$ is only $1.098(9)$ $\mathrm{MeV}$, the lowest known strength of a shell closure, as stated in Ref. [10].

\section{B. ${ }^{34} \mathrm{Al}$ : In-trap decay of ${ }^{34} \mathrm{Mg}$}

As explained above, the $1^{+}$state in ${ }^{34} \mathrm{Al}$ was populated by the decay of ${ }^{34} \mathrm{Mg}$ in the preparation trap. About $2500{ }^{34} \mathrm{Mg}$ ions were delivered by ISOLDE for each proton pulse hitting the $\mathrm{UC}_{x}$ target. However, due to the transmission efficiency of the ions from ISOLDE and due to decay losses in the RFQ buncher of ISOLTRAP (transmission efficiency lower than $1 \%$ ), only about 2 ions per proton pulse were injected into the preparation trap. Following in-trap decay for a period of $30 \mathrm{~ms}$, a recentering pulse of $5 \mathrm{~ms}$, and the following purification cycle (see Table I and Sec. II for details), the remaining ions were transported to the precision Penning trap and their cyclotron frequency was measured. The rate of ${ }^{34} \mathrm{Al}^{+}$detected on the position-sensitive detector was of about 10 events per hour. This is by far the lowest rate ever used for a ToF-ICR measurement at ISOLTRAP. Four resonances were measured, three standard ToF-ICR ones with an excitation time of $20 \mathrm{~ms}$, and one of Ramsey type with two pulses of $5 \mathrm{~ms}$ each separated by $8 \mathrm{~ms}$. Each resonance scan was performed during about $8 \mathrm{~h}$. The frequency range of the scan as well as the number of steps was decreased in order to optimize the measurement time.

Such a low count rate requires a very low noise level of the MCP. Noise measurements were performed before the run and were estimated to be on the order of 12 counts/h in a measurement time window of $300 \mu \mathrm{s}$. In order to increase the signalto-noise ratio, a position-sensitive MCP was used to clean the spectra (see Fig. 3). This detector allowed to monitor the ion position information on an event-by-event basis. Assuming that the dark counts are equally distributed on the whole surface of the MCP detector and gating on the area of the arriving ions, an important part of the background ions could be removed. Among the 12 dark counts detected per hour, only about $2 / \mathrm{h}$ were in the spatial region of interest. This feature was shown in Ref. [37] for ion-contamination cleaning. In the present case of low-statistics data, this allows to increase the signal-to-noise ratio and thus the accuracy on the center frequency. The fit of the resonance shown in the bottom part of Fig. 3 resulted in a $\chi^{2}$ of 1.03 whereas the uncleaned data fit (top panel) gave a $\chi^{2}$ of 3.3. Note that the ions do not have a pure magnetron motion when the frequency is close/at the resonance, thus the position of the ions of interest is slightly smeared out and not well defined on a spot, as seen on Fig. 3.

The fact that the MR-ToF MS could not be used during this experiment meant that the laser-ionized ${ }^{34} \mathrm{Mg}^{+}$and surface-ionized ${ }^{34} \mathrm{Al}^{+}$coming from ISOLDE, almost entirely consisting of the $4^{-}$state [17], were not mass-separated prior to the injection in the preparation trap. This separation would have been easily performed with the MR-ToF, the needed resolving power being of about 3000 . Furthermore, the short purification cycle in the preparation trap did not have the required resolving power for separating the ground and isomeric states of ${ }^{34} \mathrm{Al}^{+}$. Therefore, the beam emerging from the trap was likely a mixture of ${ }^{34} \mathrm{Al}^{+}$resulting from in-trap decay $\left(1^{+}\right.$state $)$and the one produced by ISOLDE $\left(4^{-}\right.$state). The rate of surface-ionized ${ }^{34} \mathrm{Al}^{+}$was estimated at the end of the four ToF-ICR measurements by switching off the RILIS lasers. By comparing this rate to the total rate of ${ }^{34} \mathrm{Al}^{+}$during the measurements, the percentage of $1^{+}$state was found to vary from $50(20) \%$ at the beginning of the measurements to $70(10) \%$ at the end. Nevertheless, the total ${ }^{34} \mathrm{Al}^{+}$rate was constantly increasing during the ToF-ICR measurements (due to the heating of the target during the run) and in the absence of a corresponding estimate of the surface-ionization rate, the actual weight of the ${ }^{34} \mathrm{Al}\left(1^{+}\right)$state is uncertain.

In addition, the observed rate of ${ }^{34} \mathrm{Al}^{+}$during the measurements is significantly lower than one would expect for the $1^{+}$ state considering the measured efficiency of ${ }^{34} \mathrm{Si}^{+}$in-trap production (see the next section). One important difference is that 

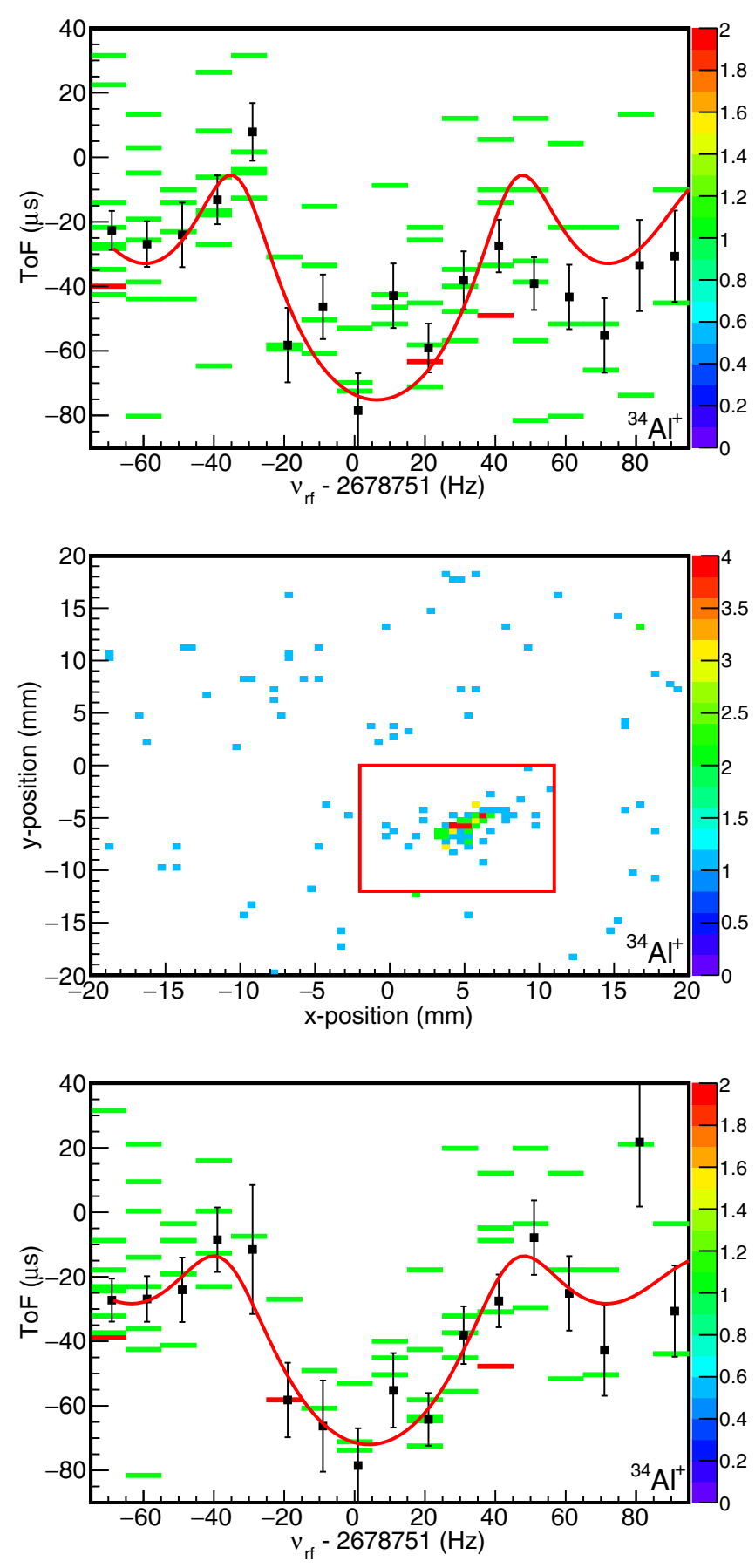

FIG. 3. ToF-ICR data of ${ }^{34} \mathrm{Al}$ produced by in-trap decay. The color-gradient scale corresponds to the number of detected ions. Top: ToF matrix and mean ToF without any position-cleaning. Middle: Positions in $x$ and $y$ of the ions hitting the MCP detector. Bottom: ToF matrix and mean ToF after the application of the position gate shown in the middle panel. The solid line is a fit of the data to the expected line-shape [30].

the axial cooling time in the case of ${ }^{34} \mathrm{Si}$ was $200 \mathrm{~ms}$, while in the case of ${ }^{34} \mathrm{Al}\left(1^{+}\right)$it was only $30 \mathrm{~ms}$. If the latter time was insufficient to cool the ${ }^{34} \mathrm{Al}\left(1^{+}\right)$ions for the subsequent recentering, the trapping efficiency of the ${ }^{34} \mathrm{Al}\left(1^{+}\right)$recoils might have been significantly smaller than in the case of ${ }^{34} \mathrm{Si}$. In addition, the decay from ${ }^{34} \mathrm{Mg}$ leads to doubly charged ${ }^{34} \mathrm{Al}$ ions. Due to the shorter cooling time for ${ }^{34} \mathrm{Al}$ there was also less time available for charge-exchange processes leading to the singly charged ${ }^{34} \mathrm{Al}$ ions investigated. In support of this idea comes the in-trap-decay production efficiency of ${ }^{33} \mathrm{Al}^{+}$from ${ }^{33} \mathrm{Mg}^{+}$, which was determined to be $5.1(6) \%$ for a $50 \mathrm{~ms}$ axial cooling time, but a smaller quadrupole recentering amplitude. This efficiency value, if applied to the production of ${ }^{34} \mathrm{Al}\left(1^{+}\right)$, would lead to an estimate of the weight of the $1^{+}$ state during the ToF-ICR measurements of about 30-50\%, so closer to what was estimated using the rate of surface-ionized ${ }^{34} \mathrm{Al}^{+}$.

All these estimates suggest that the ratio between the two ${ }^{34} \mathrm{Al}^{+}$states during the ToF-ICR measurements was on the order of 1 , but with a relatively large uncertainty, due to the absence of a direct estimate of the rate of surface-ionized ${ }^{34} \mathrm{Al}^{+}$. This estimate was not made, because only at the end of the experiment it became apparent that the surface-ionization efficiency of ${ }^{34} \mathrm{Al}^{+}$is, although very low, not negligible compared to the low efficiency of the in-trap-decay process.

If we consider the excitation energy of $46.6 \mathrm{keV}$ proposed for the $1^{+}$state in Ref. [17], which was not known during the present experiment, the short excitation time used for the ToFICR resonances could not have resolved the two states. Indeed such a low excitation energy requires a resolving power of about 700 000, corresponding to a frequency difference in the precision trap of about $3.5 \mathrm{~Hz}$. However, the presence of isomeric ions would have shifted the center frequency of the resonance notably.

The four ToF-ICR measurements result in an average mass excess value of $-2983(16) \mathrm{keV}$, which is well within one standard deviation (including the point scattering) from the mass excess of the $4^{-}$state. Considering the excitation energy of $46.6 \mathrm{keV}$ proposed for the $1^{+}$state in Ref. [17], the result deviates by more than two standard deviations from the expected mass for a pure $1^{+}$ensemble. However, the above-mentioned presence of surface-ionized ions pulls the overall average mass towards the mass of the $4^{-}$state. Indeed, the final average value determined in this work is in agreement with the expected value for a mixed ensemble, if we assume a ratio of 1 . However, the above-mentioned issues in determining this ratio with a reasonable precision makes the extraction of the excitation energy of the $1^{+}$isomer in ${ }^{34} \mathrm{Al}$ impossible.

Nevertheless, the nonobservation of a state lying at high excitation energy in the resonance allows us to confirm the report [17] that the excitation energy of this isomer is indeed much lower than the predicted $550 \mathrm{keV}$ [4]. The average mass excess value resulting from the ToF-ICR measurements also fits to the fact that the $1^{+}$state is the isomer and not the ground state in ${ }^{34} \mathrm{Al}$, in turn confirming the remarkable crossover in the nuclear mass surface. This crossover was investigated in [5] by using large-scale shell-model calculations and understood as a threshold effect associated with the entrance into the $N=20$ island of inversion. This unique feature shows how high the dominance of deformed intruder configurations in $\mathrm{Mg}$ isotopes is, compared to the $\mathrm{Al}$ isotopes with only one additional proton. 


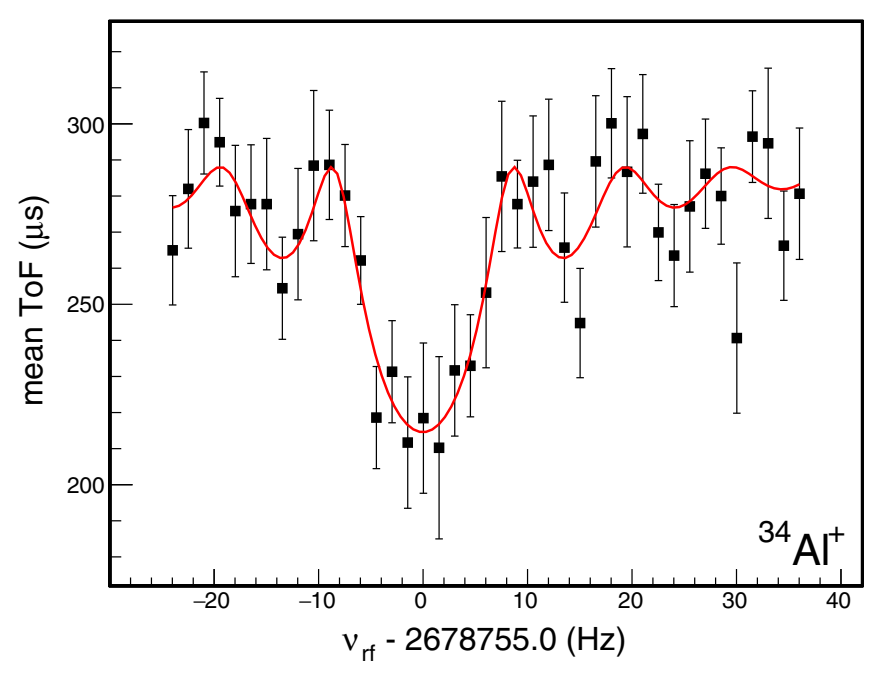

FIG. 4. ToF-ICR resonance of ${ }^{34} \mathrm{Al}^{+}$delivered by ISOLDE with an excitation time of $100 \mathrm{~ms}$. The solid curve is a fit of the data to the theoretical lineshape [30].

\section{C. ${ }^{34} \mathrm{Al}$ : Direct production by ISOLDE}

A yield of about $600{ }^{34} \mathrm{Al}$ ions/s delivered from ISOLDE was estimated. It had actually been shown in Ref. [17] that the ${ }^{34} \mathrm{Al}$ beam delivered by ISOLDE is a mixture with about $10 \%$ containing the isomeric state [38]. The half-life of the isomer being very short $(26 \mathrm{~ms})$, the ${ }^{34} \mathrm{Al}$ beam delivered to the measurement trap of ISOLTRAP would contain less than $0.5 \%$ of the isomer. In order to confirm this number, measurements were performed with different cycle times, to vary the isomeric contribution and observe either a frequency shift in the ToF-ICR resonance value or a count-rate difference due to different decay losses. However, no trace of isomer could be observed.

Two 50-ms standard ToF-ICR resonances were performed as well as one with $100 \mathrm{~ms}$ excitation time (shown in Fig. 4) and a fourth one using the Ramsey scheme with two 5-ms excitation pulses separated by a waiting period of $8 \mathrm{~ms}$. The obtained mass excess of $-2995.4(2.9) \mathrm{keV}$ is in agreement with the AME2016 value of $-3000(3) \mathrm{keV}$, which includes the result from a recent experiment performed at TITAN/TRIUMF [16]. The present result confirms the crossover observed in the two-neutron separation energies $S_{2 n}$ of ${ }^{33} \mathrm{Mg}$ and ${ }^{34} \mathrm{Al}$ [5]. As mentioned in the Introduction, this is a specific feature found nowhere else in the nuclear chart.

\section{D. ${ }^{34} \mathrm{Si}$ : Sequential in-trap decay from ${ }^{34} \mathrm{Mg}$ via ${ }^{34} \mathrm{Al}$}

${ }^{34} \mathrm{Si}$ ions were produced in the preparation trap from the two sequential $\beta$ decays ${ }^{34} \mathrm{Mg} \rightarrow{ }^{34} \mathrm{Al} \rightarrow{ }^{34} \mathrm{Si}$. Due to the relatively long half-life of ${ }^{34} \mathrm{Si}$, decay losses were low during the trap cycles and led to a count rate of about 0.2 counts/cycle on the MCP detector. A test was performed by switching the lasers off during a few minutes. Not a single ion was detected, which confirmed that the ${ }^{34} \mathrm{Si}$ isotopes were indeed produced by two sequential in-trap decays. The axial cooling time in the preparation trap was set to $200 \mathrm{~ms}$, with a $5 \mathrm{~ms}$ time for centering by a broadband quadrupole excitation. Measuring the ${ }^{34} \mathrm{Mg}^{+}$and the ${ }^{34} \mathrm{Si}^{+}$rates behind

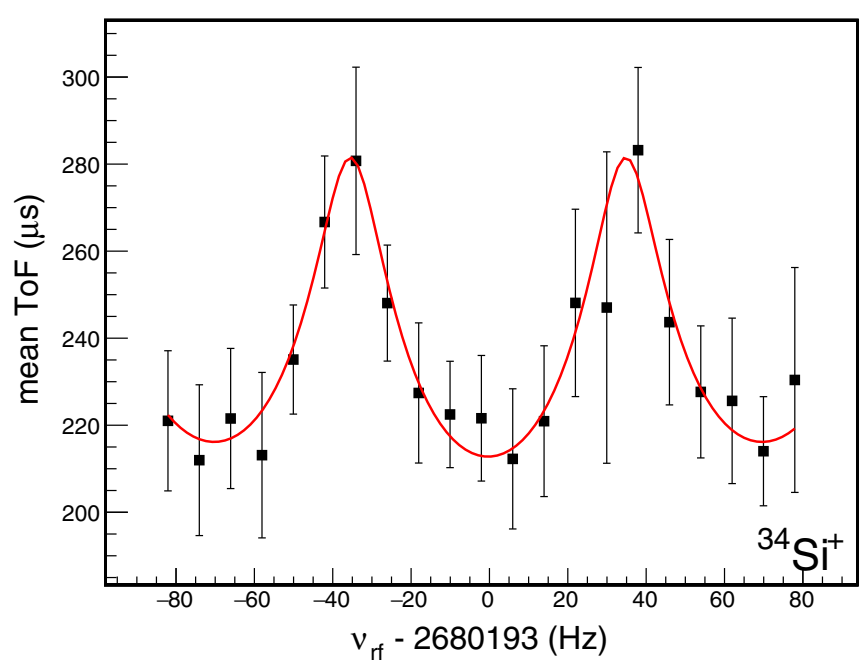

FIG. 5. Ramsey-type ToF-ICR measurement of ${ }^{34} \mathrm{Si}^{+}$. The excitation time is $18 \mathrm{~ms}$ (pulses of $5 \mathrm{~ms}$ and a waiting period of $8 \mathrm{~ms}$ ) and the number of frequency steps is 20 . The solid curve is a fit of the data to the theoretical lineshape [30].

the preparation trap and knowing the half-lives and branching ratios of the ${ }^{34} \mathrm{Mg}$ and ${ }^{34 m} \mathrm{Al}$ beta decays [17], the capture efficiency of the ${ }^{34} \mathrm{Si}^{+}$grand-daughter ions was estimated to be $20(9) \%$.

Three resonances were performed for this isotope. The quadrupole excitation in the precision trap was increased to $600 \mathrm{~ms}$ for one of the resonances, in order to increase the precision. Two other resonances were taken, one standard ToF-ICR with an excitation time of $20 \mathrm{~ms}$ and another one using the Ramsey scheme with two 5-ms excitation pulses separated by a waiting period of $8 \mathrm{~ms}$. As can be seen in Figs. 5 and 6 , the range of the frequency scan was shorter than usual as well as the number of steps decreased to 20 for the Ramsey scheme and 16 for the standard ToF-ICR resonance. These specific conditions were used to validate online the schemes used for the ${ }^{34 m} \mathrm{Al}$ measurement.

The final value of the measured mass of ${ }^{34} \mathrm{Si}$ is $-19991.7(0.8) \mathrm{keV}$ and deviates by $2.4 \sigma$ from the mass value found in the AME2016 of -19957(14) keV [4], which is the same value as the one in the AME1993 evaluation, due to the absence of newer measurements. The AME value results from transfer-reaction measurements performed between 30 and 40 years ago [39-42]. The deviation of the new value with respect to the old one is about $35 \mathrm{keV}$, which is quite small compared to the sensitivity of the nuclear models, as can be seen for example in Ref. [21]. However, this first direct mass measurement is crucial to check that this binding-energy input taken into account in the nuclear models since decades, is accurate. Furthermore, this measurement paves the way to the study of ${ }^{35,36} \mathrm{Si}$, which could be done in the future with the same recoil-ion trapping technique, and thus probe the empirical shell gap for $Z=14$.

\section{CONCLUSION}

The masses of ${ }^{34} \mathrm{Si},{ }^{34,33} \mathrm{Mg}$, and ${ }^{34} \mathrm{Al}$ were measured with the Penning-trap mass spectrometry technique. The 

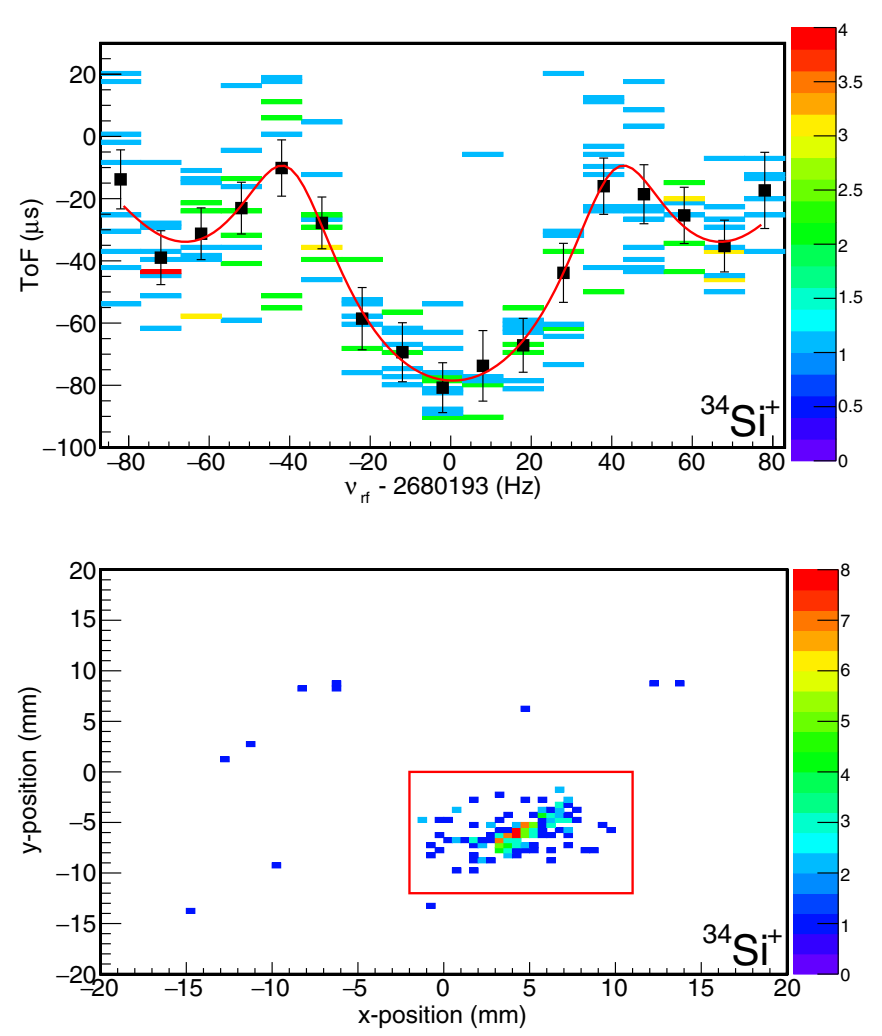

FIG. 6. ToF-ICR measurement of ${ }^{34} \mathrm{Si}^{+}$with an excitation time of $20 \mathrm{~ms}$. The color-gradient scale corresponds to the number of detected ions. Top: ToF matrix and mean ToF. The solid line is a fit of the data to the expected line-shape [30]. Bottom: Positions in $x$ and $y$ of the ions hitting the MCP detector where the red box shows the position gate. recoil-ion trapping technique allowed to show that isomeric states not populated in the target can also be produced, in this work the intruder state $1^{+}$in ${ }^{34} \mathrm{Al}$. In addition, it has been shown that a position-sensitive detector can be used to remove dark counts and thus, to increase the signal-to-noise ratio of the resonance, in particular in the case of low-statistics experiments. The new mass measurements of these isotopes close to the island of inversion allowed to confirm specific features, like the extremely low shell gap at $Z=14, N=20$ or the unique crossover of the ${ }^{33} \mathrm{Mg}$ and ${ }^{34} \mathrm{Al}$ two-neutron separation energies. Thanks to the in-trap decay technique, the refractory ${ }^{34} \mathrm{Si}$ could also be produced in an ISOL facility, and its mass was for the first time directly measured. This work paves the way to measurements further from stability, which could be performed with the PI-ICR measurement method [43-46], recently implemented with success at ISOLTRAP. Using such a method, the two states in ${ }^{34} \mathrm{Al}$ could have been separated and their masses directly measured, in addition the high sensitivity and rapidity of this technique would allow to access more exotic species.

\section{ACKNOWLEDGMENTS}

We thank the ISOLDE technical group, the ISOLDE collaboration, and the RILIS team for support. We also acknowledge support by the BMBF (05P12HGCI1, 05P12HGFNE, and 05P15ODCIA), the Max Planck Society, French IN2P3, by the Alliance Program of the Helmholtz Association Contract No. HA216/EMMI. A.W. acknowledges support by a Wolfgang Gentner Scholarship. S.K. acknowledges support from the Robert-Bosch Foundation. R.B.C. acknowledges support from the Partner Group with the Max-Planck Society.
[1] C. Thibault, R. Klapisch, C. Rigaud, A. M. Poskanzer, R. Prieels, L. Lessard, and W. Reisdorf, Direct measurement of the masses of ${ }^{11} \mathrm{Li}$ and ${ }^{26-32} \mathrm{Na}$ with an on-line mass spectrometer, Phys. Rev. C 12, 644 (1975).

[2] T. Otsuka, T. Suzuki, R. Fujimoto, H. Grawe, and Y. Akaishi, Evolution of Nuclear Shells Due to the Tensor Force, Phys. Rev. Lett. 95, 232502 (2005).

[3] O. Sorlin and M. G. Porquet, Nuclear magic numbers: New features far from stability, Prog. Part. Nucl. Phys. 61, 602 (2008).

[4] M. Wang, G. Audi, F. G. Kondev, W. J. Huang, S. Naimi, and $\mathrm{X}$. Xu, The AME2016 atomic mass evaluation, Chin. Phys. C 41, 030003 (2017).

[5] A. A. Kwiatkowski, C. Andreoiu, J. C. Bale, A. Chaudhuri, U. Chowdhury, S. Malbrunot-Ettenauer, A. T. Gallant, A. Grossheim, G. Gwinner, A. Lennarz, T. D. Macdonald, T. J. M. Rauch, B. E. Schultz, S. Seeraji, M. C. Simon, V. V. Simon, D. Lunney, A. Poves, and J. Dilling, Observation of a crossover of $S_{2 n}$ in the island of inversion from precision mass spectrometry, Phys. Rev. C 92, 061301(R) (2015).

[6] E. Caurier, F. Nowacki, and A. Poves, Merging of the islands of inversion at $N=20$ and $N=28$, Phys. Rev. C 90, 014302 (2014).
[7] J. R. Terry, B. A. Brown, C. M. Campbell, J. M. Cook, A. D. Davies, D.-C. Dinca, A. Gade, T. Glasmacher, P. G. Hansen, B. M. Sherrill, H. Zwahlen, D. Bazin, K. Yoneda, J. A. Tostevin, T. Otsuka, Y. Utsuno, and B. Pritychenko, Singleneutron knockout from intermediate energy beams of ${ }^{30,32} \mathrm{Mg}$ : Mapping the transition into the island of inversion, Phys. Rev. C 77, 014316 (2008).

[8] K. Wimmer, T. Kröll, R. Krücken, V. Bildstein, R. Gernhäuser, B. Bastin, N. Bree, J. Diriken, P. Van Duppen, M. Huyse, N. Patronis, P. Vermaelen, D. Voulot, J. Van de Walle, F. Wenander, L. M. Fraile, R. Chapman, B. Hadinia, R. Orlandi, J. F. Smith, R. Lutter, P. G. Thirolf, M. Labiche, A. Blazhev, M. Kalkühler, P. Reiter, M. Seidlitz, N. Warr, A. O. Macchiavelli, H. B. Jeppesen, E. Fiori, G. Georgiev, G. Schrieder, S. Das Gupta, G. Lo Bianco, S. Nardelli, J. Butterworth, J. Johansen, and K. Riisager, Discovery of the Shape Coexisting $0^{+}$State in ${ }^{32} \mathrm{Mg}$ by a Two Neutron Transfer Reaction, Phys. Rev. Lett. 105, 252501 (2010).

[9] G. Neyens, Multiparticle-multihole states in ${ }^{31} \mathrm{Mg}$ and ${ }^{33} \mathrm{Mg}$ : A critical evaluation, Phys. Rev. C 84, 064310 (2011).

[10] A. Chaudhuri, C. Andreoiu, T. Brunner, U. Chowdhury, S. Ettenauer, A. T. Gallant, G. Gwinner, A. A. Kwiatkowski, A. Lennarz, D. Lunney, T. D. Macdonald, B. E. Schultz, 
M. C. Simon, V. V. Simon, and J. Dilling, Evidence for the extinction of the $N=20$ neutron-shell closure for ${ }^{32} \mathrm{Mg}$ from direct mass measurements, Phys. Rev. C 88, 054317 (2013).

[11] F. Rotaru, F. Negoita, S. Grévy, J. Mrazek, S. Lukyanov, F. Nowacki, A. Poves, O. Sorlin, C. Borcea, R. Borcea, A. Buta, L. Cáceres, S. Calinescu, R. Chevrier, Zs. Dombrádi, J. M. Daugas, D. Lebhertz, Y. Penionzhkevich, C. Petrone, D. Sohler, M. Stanoiu, and J. C. Thomas, Unveiling the Intruder Deformed $0^{+}$State in ${ }^{34} \mathrm{Si}$, Phys. Rev. Lett. 109, 092503 (2012).

[12] P. Himpe, G. Neyens, D. L. Balabanski, G. Bélier, D. Borremans, J. M. Daugas, F. de Oliveira Santos, M. De Rydt, K. Flanagan, G. Georgiev, M. Kowalska, S. Mallion, I. Matea, P. Morel, Yu. E. Penionzhkevich, N. A. Smirnova, C. Stodel, K. Turzó, N. Vermeulen, and D. Yordanov, $g$ factors of ${ }^{31,32,33} \mathrm{Al}$ : Indication for intruder configurations in the ${ }^{33} \mathrm{Al}$ ground state, Phys. Lett. B 643, 257 (2006).

[13] P. Himpe, G. Neyens, D. L. Balabanski, G. Bélier, J. M. Daugas, F. de Oliveira Santos, M. De Rydt, K. T. Flanagan, I. Matea, P. Morel, Yu. E. Penionzhkevich, L. Perrot, N. A. Smirnova, C. Stodel, J. C. Thomas, N. Vermeulen, D. T. Yordanov, Y. Utsuno, and T. Otsuka, $g$ factor of the exotic $N=21$ isotope ${ }^{34} \mathrm{Al}$ : probing the $N=20$ and $N=28$ shell gaps at the border of the island of inversion, Phys. Lett. B 658, 203 (2008).

[14] H. Heylen, M. De Rydt, G. Neyens, M. L. Bissell, L. Caceres, R. Chevrier, J. M. Daugas, Y. Ichikawa, Y. Ishibashi, O. Kamalou, T. J. Mertzimekis, P. Morel, J. Papuga, A. Poves, M. M. Rajabali, C. Stödel, J. C. Thomas, H. Ueno, Y. Utsuno, N. Yoshida, and A. Yoshimi, High-precision quadrupole moment reveals significant intruder component in ${ }_{13}^{33} \mathrm{Al}_{20}$ ground state, Phys. Rev. C 94, 034312 (2016).

[15] Z. Y. Xu, H. Heylen, K. Asahi, F. Boulay, J. M. Daugas, R. P. de Groote, W. Gins, O. Kamalou, Á. Koszorús, M. Lykiardopoulou, T. J. Mertzimekis, G. Neyens, H. Nishibata, T. Otsuka, R. Orset, A. Poves, T. Sato, C. Stodel, J. C. Thomas, N. Tsunoda, Y. Utsuno, M. Vandebrouck, and X. F. Yang, Nuclear moments of the low-lying isomeric $1^{+}$state of ${ }^{34} \mathrm{Al}$ : Investigation on the neutron $1 p 1 h$ excitation across $N=20$ in the island of inversion, Phys. Lett. B 782, 619 (2018).

[16] A. T. Gallant, M. Alanssari, J. C. Bale, C. Andreoiu, B. R. Barquest, U. Chowdhury, J. Even, A. Finlay, D. Frekers, G. Gwinner, R. Klawitter, B. Kootte, A. A. Kwiatkowski, D. Lascar, K. G. Leach, E. Leistenschneider, A. Lennarz, A. J. Mayer, D. Short, R. Thompson, M. Wieser, D. Lunney, and J. Dilling, Mass determination near $N=20$ for $\mathrm{Al}$ and $\mathrm{Na}$ isotopes, Phys. Rev. C 96, 024325 (2017).

[17] R. Lica, F. Rotaru, M. J. G. Borge, S. Grevy, F. Negoita, A. Poves, O. Sorlin, A. N. Andreyev, R. Borcea, C. Costache, H. DeWitte, L. M. Fraile, P. T. Greenlees, M. Huyse, A. Ionescu, S. Kisyov, J. Konki, I. Lazarus, M. Madurga, N. Marginean, R. Marginean, C. Mihai, R. E. Mihai, A. Negret, R. D. Page, J. Pakarinen, S. Pascu, V. Pucknell, P. Rahkila, E. Rapisarda, A. Serban, C. O. Sotty, L. Stan, M. Stanoiu, O. Tengblad, A. Turturica, P. VanDuppen, R. Wadsworth, and N. Warr, Identification of the crossing point at $N=21$ between normal and intruder configurations, Phys. Rev. C 95, 021301(R) (2017).

[18] P. Baumann, A. Huck, G. Klotz, A. Knipper, G. Walter, G. Marguier, H. L. Ravn, C. Richard-Serre, A. Poves, and J. Retamosa, ${ }^{34} \mathrm{Si}$ : A new doubly magic nucleus? Phys. Lett. B 228, 458 (1989).
[19] S. Nummela, P. Baumann, E. Caurier, P. Dessagne, A. Jokinen, A. Knipper, G. LeScornet, C. Miehe, F. Nowacki, M. Oinonen, Z. Radivojevic, M. Ramdhane, G. Walter, and J. Aysto (ISOLDE Collaboration), Spectroscopy of ${ }^{34,35} \mathrm{Si}$ by $\beta$ decay: $s d-f p$ shell gap and single-particle states, Phys. Rev. C 63, 044316 (2001).

[20] A. Mutschler, A. Lemasson, O. Sorlin, D. Bazin, C. Borcea, R. Borcea, Z. Dombrádi, J. P. Ebran, A. Gade, H. Iwasaki, E. Khan, A. Lepailleur, F. Recchia, T. Roger, F. Rotaru, D. Sohler, M. Stanoiu, S. R. Stroberg, J. A. Tostevin, M. Vandebrouck, D. Weisshaar, and K. Wimmer, A proton density bubble in the doubly magic ${ }^{34}$ Si nucleus, Nat. Phys. 13, 152 (2017).

[21] T. Duguet, V. Somà, S. Lecluse, C. Barbieri, and P. Navrátil, $A b$ initio calculation of the potential bubble nucleus ${ }^{34} \mathrm{Si}$, Phys. Rev. C 95, 034319 (2017).

[22] A. Herlert, D. Beck, K. Blaum, F. Carrel, P. Delahaye, S. George, C. Guénaut, F. Herfurth, A. Kellerbauer, H.-J. Kluge, D. Lunney, M. Mukherjee, L. Schweikhard, and C. Yazidjian, Mass spectrometry of atomic ions produced by in-trap decay of short-lived nuclides, New J. Phys. 7, 44 (2005).

[23] A. Herlert, S. Van Gorp, D. Beck, K. Blaum, M. Breitenfeldt, R. B. Cakirli, S. George, U. Hager, F. Herfurth, A. Kellerbauer, D. Lunney, R. Savreux, L. Schweikhard, and C. Yazidjian, Recoil-ion trapping for precision mass measurements, Eur. Phys. J. A 48, 97 (2012).

[24] F. Rotaru, R. Lica, F. Negoita, S. Grévy, N. Marginean, Ph. Dessagne, T. Stora, C. Borcea, R. Borcea, S. Calinescu, J. M. Daugas, D. Filipescu, I. Kuti, L. M Fraille, S. Franchoo, I. Gheorghe, R. Marginean, C. Mihai, P. Morfouace, J. Mrazek, P. Morel, A. Negret, D. Pietreanu, T. Sava, D. Sohler, M. Stanoiu, I. Stefan, R. Suvaila, and S. Toma, Properties of intruder states in ${ }^{34} \mathrm{Al}$ and ${ }^{34} \mathrm{Si}$, AIP Conf. Proc. 1645, 363 (2015).

[25] M. Mukherjee, D. Beck, K. Blaum, G. Bollen, J. Dilling, S. George, F. Herfurth, A. Herlert, A. Kellerbauer, H. J. Kluge, S. Schwarz, L. Schweikhard, and C. Yazidjian, ISOLTRAP: An on-line Penning trap for mass spectrometry on short-lived nuclides, Eur. Phys. J. A 35, 1 (2008).

[26] S. Rothe, T. Day Goodacre, D. V. Fedorov, V. N. Fedosseev, B. A. Marsh, P. L. Molkanov, R. E. Rossel, M. D. Seliverstov, M. Veinhard, and K. D. A. Wendt, Laser ion beam production at CERN-ISOLDE: New features - More possibilities, Nucl. Instrum. Methods Phys. Res. B 376, 91 (2016).

[27] S. Kreim, D. Atanasov, D. Beck, K. Blaum, Ch. Böhm, Ch. Borgmann, M. Breitenfeldt, T. E. Cocolios, D. Fink, S. George, A. Herlert, A. Kellerbauer, U. Köster, M. Kowalska, D. Lunney, V. Manea, E. Minaya Ramirez, S. Naimi, D. Neidherr, T. Nicol, R. E. Rossel, M. Rosenbusch, L. Schweikhard, J. Stanja, F. Wienholtz, R. N. Wolf, and K. Zuber, Recent exploits of the ISOLTRAP mass spectrometer Nucl. Instrum. Methods Phys. Res. B 317, 492 (2013).

[28] R. N. Wolf, F. Wienholtz, D. Atanasov, D. Beck, K. Blaum, Ch. Borgmann, F. Herfurth, M. Kowalska, S. Kreim, Yu. A. Litvinov, D. Lunney, V. Manea, D. Neidherr, M. Rosenbusch, L. Schweikhard, J. Stanja, and K. Zuber, ISOLTRAP's multireflection time-of-flight mass separator/spectrometer, Int. J Mass Spectrom. 349-350, 123 (2013).

[29] G. Bollen, R. B. Moore, G. Savard, and H. Stolzenberg, The accuracy of heavy-ion mass measurements using time of flightion cyclotron resonance in a Penning trap, J. Appl. Phys. 68, 4355 (1990). 
[30] M. König, G. Bollen, H.-J. Kluge, T. Otto, and J. Szerypo, Quadrupole excitation of stored ion motion at the true cyclotron frequency, Int. J. Mass Spectrom. 142, 95 (1995).

[31] R. N. Wolf, D. Beck, K. Blaum, Ch. Böhm, Ch. Borgmann, M. Breitenfeldt, N. Chamel, S. Goriely, F. Herfurth, M. Kowalska, S. Kreim, D. Lunney, V. Manea, E. Minaya Ramirez, S. Naimi, D. Neidherr, M. Rosenbusch, L. Schweikhard, J. Stanja, F. Wienholtz, and K. Zuber, Plumbing Neutron Stars to New Depths with the Binding Energy of the Exotic Nuclide ${ }^{82} \mathrm{Zn}$, Phys. Rev. Lett. 110, 041101 (2013).

[32] F. Wienholtz, D. Beck, K. Blaum, Ch. Borgmann, M. Breitenfeldt, R. B. Cakirli, S. George, F. Herfurth, J. D. Holt, M. Kowalska, S. Kreim, D. Lunney, V. Manea, J. Menéndez, D. Neidherr, M. Rosenbusch, L. Schweikhard, A. Schwenk, J. Simonis, J. Stanja, R. N. Wolf, and K. Zuber, Masses of exotic calcium isotopes pin down nuclear forces, Nature 498, 346 (2013).

[33] G. Savard, St. Becker, G. Bollen, H.-J. Kluge, R. B. Moore, Th. Otto, L. Schweikhard, H. Stolzenberg, and U. Wiess, A new cooling technique for heavy ions in a Penning trap, Phys. Lett. A 158, 247 (1991).

[34] S. Van Gorp, M. Beck, M. Breitenfeldt, V. De Leebeeck, P. Friedag, A. Herlert, T. Iitaka, J. Mader, V. Kozlov, S. Roccia, G. Soti, M. Tandecki, E. Traykov, F. Wauters, Ch. Weinheimer, D. Zákoucký, and N. Severijns, Simbuca, using a graphics card to simulate Coulomb interactions in a Penning trap, Nucl. Instrum. Methods Phys. Res. A 638, 192 (2011).

[35] A. Kellerbauer, K. Blaum, G. Bollen, F. Herfurth, H.-J. Kluge, M. Kuckein, E. Sauvan, C. Scheidenberger, and L. Schweikhard, From direct to absolute mass measurements: A study of the accuracy of ISOLTRAP, Eur. Phys. J. D 22, 53 (2003).

[36] B. Jurado, H. Savajols, W. Mittig, N. A. Orr, P. RousselChomaz, D. Baiborodin, W. N. Catford, M. Chartier, C. E. Demonchy, Z. Dlouhý, A. Gillibert, L. Giot, A. Khouaja, A. Lépine-Szily, S. Lukyanov, J. Mrazek, Y. E. Penionzhkevich, S. Pita, M. Rousseau, and A. C. Villari, Mass measurements of neutron-rich nuclei near the $N=20$ and 28 shell closures, Phys. Lett. B 649, 43 (2007).

[37] J. Ketelaer, K. Blaum, M. Block, K. Eberhardt, M. Eibach, R. Ferrer, S. George, F. Herfurth, J. Ketter, Sz. Nagy, J. Repp,
L. Schweikhard, C. Smorra, S. Sturm, and S. Ulmer, Recent developments in ion detection techniques for Penning trap mass spectrometry at TRIGA-TRAP, Eur. Phys. J. A 42, 311 (2009).

[38] R. Lica (private communication).

[39] A. M. Nathan and D. E. Alburger, Mass and beta decay of ${ }^{34} \mathrm{Si}$, Phys. Rev. C 15, 1448 (1977).

[40] W. A. Mayer, W. Henning, R. Holzwarth, H. J. Körner, G. Korschinek, G. Rosner, and H. J. Scheerer, Mass excess and excited states of neutron-rich silicon, phosphorus and sulphur isotopes, Z. Phys. A 319, 287 (1984).

[41] L. K. Fifield, C. L. Woods, R. A. Bark, P. V. Drumm, and M. A. C. Hotchkis, Masses and level schemes of ${ }^{33} \mathrm{Si}$ and ${ }^{34} \mathrm{Si}$, Nucl. Phys. A 440, 531 (1984).

[42] P. J. Woods, R. Chapman, J. L. Durell, J. N. Mo, R. J. Smith, N. E. Sanderson, B. R. Fulton, and R. A. Cunningham, Use of the ${ }^{64} \mathrm{Ni}\left({ }^{36} \mathrm{~S},{ }^{34} \mathrm{Si}\right){ }^{66} \mathrm{Zn}$ reaction to measure the mass of ${ }^{34} \mathrm{Si}$, Z. Phys. J. A 321, 119 (1985).

[43] S. Eliseev, K. Blaum, M. Block, C. Droese, M. Goncharov, E. Minaya Ramirez, D. A. Nesterenko, Yu. N. Novikov, and L. Schweikhard, Phase-Imaging Ion-Cyclotron-Resonance Measurements for Short-Lived Nuclides, Phys. Rev. Lett. 110, 082501 (2013).

[44] S. Eliseev, K. Blaum, M. Block, A. Dörr, C. Droese, T. Eronen, M. Goncharov, M. Höcker, J. Ketter, E. Minaya Ramirez, D. A. Nesterenko, Yu. N. Novikov, and L. Schweikhard, A phase-imaging technique for cyclotron-frequency measurements, Appl. Phys. B 114, 107 (2014).

[45] D. A. Nesterenko, T. Eronen, A. Kankainen, L. Canete, A. Jokinen, I. D. Moore, H. Penttilä, S. Rinta-Antila, and A. de Roubin, Phase-imaging ion-cyclotron-resonance technique at the JYFLTRAP double Penning trap mass spectrometer, Eur. Phys. J. A 54, 154 (2018).

[46] R. Orford, N. Vassh, J. A. Clark, G. C. McLaughlin, M. R. Mumpower, G. Savard, R. Surman, A. Aprahamian, F. Buchinger, M. T. Burkey, D. A. Gorelov, T. Y. Hirsh, J. W. Klimes, G. E. Morgan, A. Nystrom, and K. S. Sharma, Precision Mass Measurements of Neutron-Rich Neodymium and Samarium Isotopes and Their Role in Understanding Rare-Earth Peak Formation, Phys. Rev. Lett. 120, 262702 (2018). 